\title{
Disputas políticas locales e instituciones binacionales en la zona fronteriza de Ecuador y Colombia
}

\author{
Santiago Carranco Paredes, Éricka Poveda Lugo, Angie Arias Burgos
}

Recepción: 3 de septiembre de 2019

Aprobación: 30 de Octubre de 2019

\begin{abstract}
Resumen
Este artículo pretende analizar la construcción del Plan Binacional de Integración Fronteriza de Ecuador y Colombia (PBIFEC) como una institución binacional, la que se constituyó gracias a un esfuerzo en conjunto de funcionarios gubernamentales de ambos estados. A su vez, demuestra que los resultados que dicha institución ha mantenido dentro de la Zona de Integración Fronteriza de Ecuador y Colombia (ZIFEC) han sido limitados. En este sentido, se ofrece una explicación de los motivos por los cuales los esfuerzos gubernamentales han sido nulos en la zona, los que se encuentran relacionados a la poca presencia estatal que se ha mantenido históricamente en los territorios de la ZIFEC. Esto ha generado que las dinámicas sociales se configuren a partir de instituciones informales consolidadas por medio de la costumbre.

\section{Palabras Clave}

Instituciones Binacionales - Instituciones Informales - Territorios Fronterizos Cooperación Internacional - Disputas Locales.

\section{Absract}

This text aims to analyse the consolidation of the Plan Binacional de Integración Fronteriza de Ecuador y Colombia (PBIFEC) as a binational institution. In order to achieve this, it was required a joint effort of government officials from both states. At the same time, this article demonstrates the limited impact that this institution has made within the Zona de Integración Fronteriza de Ecuador y Colombia (ZIFEC). In this connection, this work offers an explanation of the failure that the government efforts have had in that zone, related to the little state presence that has historically been maintained in the ZIFEC territories, which has generated social dynamics that have been configurated by informal institutions consolidated through custom.

\section{Key Words}

Binational Institutions - Informal Institutions - Frontier Territories - International Cooperation - Local Disputes.
\end{abstract}




\section{Introducción}

$\longrightarrow 1$ plan binacional de integración fronteriza (PBIFEC) surge dentro de un marco de ideas que pretende instrumentalizar a la cooperación internacional con el fin de generar desarrollo en las zonas de frontera. A su vez, el PBIFEC se genera como un producto del esfuerzo gubernamental entre Ecuador y Colombia con el objetivo de acarrear problemas singulares y conjuntos dentro de la zona en donde mantiene efecto dicho instrumento (Zona de integración Fronteriza Ecuador-Colombia ZIFEC). Lo interesante de este proceso es que se crea justamente para llegar por medio de una institución binacional a zonas fronterizas caracterizadas históricamente por ser espacios en donde ha existido poca presencia estatal.

En este sentido, un esfuerzo desde arriba (estados-sociedades), por medio de una institución binacional, puede no tener el resultado deseado en una zona en donde la presencia estatal ha sido débil. Esto debido a varios factores como la presencia de instituciones informales que se encuentran arraigadas por medio de la costumbre (Acemoglu y Robinson 2017), los incentivos económicos de grupos que se benefician al estar fuera de las regulaciones estatales y la aversión al riesgo que pueden tener las poblaciones hacia una injerencia externa de un Estado que se lo ha considerado como lejano (Moravcsik 2012).

De esta manera, el presente trabajo pretende analizar, desde varias perspectivas, la política de integración fronteriza de Ecuador y Colombia, así como su proceso de construcción, implementación y resultados. Para este cometido, se parte de los conceptos de cooperación para el desarrollo, instituciones, zonas de frontera y configuración de preferencias societales para poder acercarse a las nociones sobre el fenómeno en cuestión. Mientras que por medio de entrevistas a funcionarios gubernamentales se comprenderá la forma en la que se generó la institución binacional y la manera en la que se homogenizaron los intereses de ambos estados; mediante la utilización de estadística descriptiva se analizará los resultados generales de dichas políticas. Asimismo, por medio de entrevistas a funcionarios que se encuentran en el territorio de la ZIFEC se comprenderá las razones por las cuales se ha dificultado la implementación del PBIFEC dentro de la zona fronteriza. 


\section{Metodología}

Este trabajo trata de dar respuesta directa a las razones por las cuales el PBIFEC no ha logrado sostener los resultados deseados dentro de la ZIFEC. Si bien el neoliberalismo institucional ha propuesto que es posible modificar conductas estatales a través de instituciones transnacionales (Robert y Keohane 1985; Keohane 1988), en el caso del PBIFEC se ha visualizado que este postulado se cumple tan solo de manera parcial. En efecto, se ha logrado armonizar las nociones de desarrollo y los ejes para poder alcanzar dicho desarrollo en las zonas de frontera de dos gobiernos centrales (ColombiaEcuador). Sin embargo, cuando se trata de percibir el funcionamiento que debería mantener el PBIFEC dentro de la ZIFEC, la existencia y los resultados de dicha institución binacional siguen siendo nulos.

Por esta razón, el presente trabajo fundó su obtención de datos de carácter cualitativo por medio de documentación oficial y entrevistas semiestructuradas a funcionarios que se involucraron en el proceso de generación del PBIFEC. A su vez, se contrastó dicha información con la obtenida por medio de entrevistas semiestructuradas y la realización de grupos focales a funcionarios que se encuentran trabajando dentro del territorio de la ZIFEC. Esto se lo realizó para poder comprender las divergencias entre la manera en la que se construye y se implementa la noción de desarrollo desde arriba y como se la percibe y se ejecuta dentro del territorio.

La muestra de entrevistados $(\mathrm{N}=4)$ se las obtuvo mediante un muestreo no lineal de bola de nieve, en donde se mantuvo dos entradas. La primera, por medio de funcionarios de la cancillería ecuatoriana y la segunda, por medio de funcionarios de un organismo internacional que presta atención humanitaria en la ZIFEC. En el primer grupo se realizaron entrevistas semiestructuradas para lograr recabar información acerca de cómo se construyeron, de manera conjunta, entre funcionarios ecuatorianos y colombianos, las ideas de desarrollo deseable para implementar en la ZIFEC. Mientras que dentro del segundo grupo, se realizaron grupos focales $(\mathrm{N}=7)$ para lograr capturar el sentido por el cual se dificulta la implementación del PBIFEC dentro de la ZIFEC y la disociación que las instituciones estatales mantienen con la realidad social de dichas zonas. 


\section{Tabla 1:}

Codificación y descripción de expertos entrevistados

\begin{tabular}{|l|l|}
\hline Código & \multicolumn{1}{c|}{ Descripción del entrevistado } \\
\hline E1 & Diplomático de carrera experto en relaciones vecinales. \\
\hline E2 & Consultor político experto en zonas fronterizas. \\
\hline E3 & Diplomático de carrera experto en relaciones vecinales. \\
\hline E4 & Consultor internacional experto en integración fronteriza. \\
\hline F1 & $\begin{array}{l}\text { Grupo focal realizado con la participación de } 7 \text { miembros de una organización } \\
\text { humanitaria no gubernamental que trabaja en los territorios de la ZIFEC. }\end{array}$ \\
\hline
\end{tabular}

Tabla elaborada por el autor.

Por otra parte, se tomaron datos de los macro indicadores correspondientes a los ejes de desarrollo del PBIFEC (Tabla 2), para lograr visualizar por medio de estadística descriptiva los resultados de PBIFEC desde su implementación hasta el año 2017. Esto se hizo para poder corroborar por medio de datos oficiales la manera en la que las políticas binacionales han impactado en el desarrollo de la zona en temas de seguridad, desarrollo productivo e inclusión social. Los indicadores utilizados para visualizar el impacto de PBIFEC en la ZIFEC fueron el coeficiente de Gini, la tasa de nacimientos en mujeres entre 15-19 años, la tasa de homicidio con armas de fuego y datos relativos a la ocupación plena y precarizada.

\section{Marco Conceptual}

Cooperación para el desarrollo

Al hablar de desarrollo dentro de las zonas de frontera, se hace referencia a un sentido multidimensional como bien se lo define dentro del PBIFEC (Tabla 2 ). En este sentido, existen varios ejes propuestos para lograr un desarrollo integral comprendidos en objetivos como la eliminación de la pobreza, mejoramiento de la infraestructura pública, implementación de servicios 
básicos, integración regional y en general, la implementación de un espacio en donde se puedan asegurar los derechos básicos para una comunidad (Senplades-DNP 2014).

\section{Tabla 2:}

Indicadores asociados con los ejes de intervención y concepción de desarrollo del PBIFEC.

\begin{tabular}{|l|l|l|}
\hline \multicolumn{1}{|c|}{ Eje de intervención } & \multicolumn{1}{|c|}{ Variable } & \multicolumn{1}{c|}{ Indicadores } \\
\hline \multirow{3}{*}{ Social y cultural } & \multicolumn{1}{|c|}{$\begin{array}{c}\text { Desigualdad de } \\
\text { ingresos }\end{array}$} & \multicolumn{1}{c|}{ Coeficiente de Gini } \\
\cline { 2 - 3 } & Embarazos adolescentes & $\begin{array}{l}\text { Nacimientos en mujeres entre 15-19 } \\
\text { años. }\end{array}$ \\
\cline { 2 - 3 } & Educación básica & Tasa de analfabetismo \\
\hline \multirow{2}{*}{ Seguridad y defensa } & Muertes violentas & Tasa de homicidio a mujeres. \\
\cline { 2 - 3 } & $\begin{array}{l}\text { Tasa de homicidio con armas de } \\
\text { fuego }\end{array}$ \\
\hline \multirow{2}{*}{$\begin{array}{l}\text { Desarrollo económico y } \\
\text { productivo }\end{array}$} & Crecimiento económico & Variación del PIB \\
\cline { 2 - 3 } & Desempleo & Tasa de ocupación \\
\cline { 2 - 3 } & & Tasa de subempleo \\
\hline
\end{tabular}

Tabla elaborada por el autor a partir de los datos de Plan Binacional de Información (2014)

En la introducción del documento del Plan Binacional de Integración Fronteriza entre Ecuador y Colombia (PBIFEC), se puede observar que ambos estados instauran este instrumento con el fin de implementar un proyecto en conjunto que permita que Ecuador y Colombia se encaminen hacia el Buen Vivir y la Prosperidad. Posteriormente, el término desarrollo aparece en varios apartados y es el eje conductor del plan.

Como esfuerzo de planificación conjunta entre ambos países, se ha tratado de implementar políticas y estrategias de crecimiento binacional que permitan alcanzar objetivos comunes de desarrollo. De acuerdo con el PBIFEC, se inicia la elaboración de instrumentos normativos que permitan a los dos países dar prioridad y establecer lineamientos para el desarrollo de sus regiones transfronterizas (Senplades-DNP 2014). De esta manera, las relaciones bilaterales que conformaron Colombia y Ecuador fueron con la 
finalidad de complementar capacidades nacionales pretendiendo mejorar la calidad de vida de su población.

El plan a su vez se enmarca en un esfuerzo de cooperación binacional para el desarrollo. La cooperación internacional es una actividad de dimensión global que realizan entre sí gobiernos nacionales, subnacionales, organismos multilaterales, organizaciones de la sociedad civil, fundaciones y universidades (Prado Lallande 2016). Por medio de acciones colectivas entre dos o más miembros de la sociedad internacional, la cooperación para el desarrollo tiene el objetivo de incentivar factores acordes al bienestar y sostenibilidad. En la actualidad, la cooperación internacional para el desarrollo ha ido incrementando su apertura dentro de la política exterior, y uno de sus principales preceptos es que contribuye al desarrollo nacional y, según el caso, puede ser de alcance mundial, regional, nacional y local. De este modo, para concretar la cooperación, en este caso entre dos Estados, se materializa a través de instrumentos, destacando la cooperación técnica, la científica, la tecnológica, la cultural, la económica, la deportiva y la asistencia humanitaria (Prado Lallande 2016).

Desde una perspectiva operativa, la cooperación internacional para el desarrollo se instrumenta por la vía de proyectos (acciones puntuales), programas (conjunto de proyectos) o incluso recurriendo a cambios sistémicos en determinadas políticas públicas en ciertos países (como reformas económicas estructurales en países en desarrollo) (Prado Lallande 2016).

De acuerdo con el Plan Binacional de Integración Fronteriza entre Ecuador y Colombia, ambos deciden crear este instrumento para promover el desarrollo en una zona que fue abandonada y no tuvo una gran influencia estatal. Con esto, se establece que las instituciones tienen la capacidad de cambiar el comportamiento de los Estados y también se da la presencia, claramente, de la cooperación internacional para el desarrollo.

Teóricamente, se puede promover la cooperación internacional bajo el uso de instituciones. No obstante, los actores racionales comprenden que el comienzo de un proceso de cooperación puede constituirse como algo muy difícil. Por ello, la función de las instituciones es trabajar como medio para que se diseñen o se superen obstáculos entre los diferentes actores y se pueda 
lograr la cooperación internacional (Dai, et al. 2017).

Ahora bien, conforme al Plan de Integración Fronteriza entre Ecuador y Colombia, se establece que se construye una red institucional con el fin de permitir y establecer lineamientos que posibiliten el desarrollo en la zona fronteriza. De la misma manera, se atribuye al instrumento un carácter técnico, que, en términos de cooperación internacional para el desarrollo, es la modalidad más recurrida por los múltiples organismos internacionales e instituciones nacionales, y consiste en la transferencia de conocimientos, habilidades y experiencias entre dos o más agentes de la sociedad internacional (Prado Lallande 2016).

Por su parte, el entrevistado E1 menciona que los indicios para hablar de desarrollo en las fronteras comienzan a partir de la elaboración del Plan Ecuador. Sin embargo, en el caso de Colombia, se habló mucho antes de desarrollo en las fronteras y se implementaron planes a partir de ello. Con el Plan Binacional de Integración Fronteriza, cada obra enfocada a infraestructura, salud, educación entre otros temas, corresponde a un avance en el desarrollo de las zonas de frontera enmarcados en temáticas multidimensionales.

Por otro lado, en el F7 se mencionó que los planes de desarrollo se ven más en función de la misma comunidad y no se ve realmente a la participación del Estado. Por un lado, las entidades de mayor predominancia en la zona han sido el Ministerio de Relaciones Exteriores y la Defensoría del Pueblo. Desde el aspecto social, se ha podido connotar el esfuerzo institucional en promover el desarrollo bajo estas dos entidades, sin embargo, la comunidad en sí se preocupa más y trata de promover su desarrollo en base a instituciones informales que ellos mismos generan, ya que les ofrecen beneficios más palpables y de corto plazo, como la seguridad de estar dentro de un grupo subversivo o la renta producida por actividades relacionadas al contrabando.

\section{Desarrollo Multidimensional}

Se puede hablar de desarrollo multidimensional y de la cooperación al referirnos al desarrollo en transición. De acuerdo con la CEPAL (2018), el enfoque multidimensional respecto de la cooperación internacional para 
el desarrollo requiere la adopción de políticas eficaces para hacer frente a los retos estructurales nuevos y persistentes. Con ello, la cooperación internacional para el desarrollo debe medir el desarrollo más allá del PIB per cápita; las estrategias de cooperación y la orientación del desarrollo deben vincularse a estrategias nacionales y reflejar un enfoque multidimensional.

La cooperación internacional para el desarrollo debe ir más allá de las modalidades tradicionales. Los enfoques específicos permitirían a algunos países beneficiarse de instrumentos ad hoc para apoyar la movilización de recursos internos y otras prioridades de política, fundamentales para la transición hacia mayores niveles de desarrollo. Entre esos instrumentos figura el desarrollo de capacidades mediante el análisis de pares, u otro tipo de intercambio similar entre instituciones, así como modalidades innovadoras de intercambio de conocimientos y transferencia tecnológica (CEPAL 2018).

\section{Zonas de frontera e integración fronteriza}

Existen varios autores que han tratado de teorizar sobre las zonas de frontera desde varias perspectivas. En términos netamente jurídicos basados en la idea de soberanía internacional, se mantiene aún la concepción de Hugo Grocio, quien expresó una definición de fronteras en su obra De Jure Belli ac Pacis, en la que las detalla como una línea de separación, la que puede ser establecida por marcas de distinción. No obstante, esta leve definición de fronteras nace como un concepto que sirva para fijar o delimitar la jurisdicción que tiene un Estado sobre su territorio.

De manera imperativa se debe hacer una aclaración y una distinción entre fronteras y límites. Por un lado, de acuerdo con Lucius Caflisch, en su obra A Typology of Borders, nos ofrece el concepto de fronteras como una línea que separa los territorios terrestres sobre los que los Estados ejercen soberanía total (Caflisch 2010). En contraste, los límites estarían definidos como líneas que dividen, es decir, son espacios en los que los Estados no ejercen jurisdicción territorial plena, como los espacios marítimos que pertenezcan al mismo estado o a diferentes Estados, o las líneas que separen dichos espacios nacionales de las zonas internacionales (Caflisch 2010). 


\section{DISPUTAS POLIITICAS LOCALES E INSTITUCIONES BINACIONALES EN LA ZONA FRONTERIZA DE ECUADOR Y COLOMBIA}

Pese a ello, la definición de fronteras ha ido transformándose. Actualmente, se puede tener dos concepciones acerca de este término: frontera como zona o zona de frontera; y frontera como límite. La frontera como zona, o zona de frontera es, de acuerdo con Seara Vásquez, un área entre los territorios de dos Estados, de extensión variable y con un régimen no muy definido (Seara 2004). En este sentido, la frontera de Ecuador y Colombia no se encuentra definida por los varios procesos económicos, sociales, políticos, culturales y familiares que suceden dentro del espacio fronterizo (Carrión 2013).

Dentro de esta línea, Matiuzzi de Souza (2018) analiza en diferentes etapas académicas y empíricas como las nociones de bordes se han visto afectadas por las concepciones epistemológicas que han dominado los estudios sobre integración regional en cada una de dichas etapas. La primera etapa, conocida como el viejo regionalismo, entendía la noción de bordes como una línea divisoria entre territorios, la cual definía hasta donde un estado podía ejercer su poder y control, muy parecido a la concepción grotiana jurídica. Si bien la noción de bordes del viejo regionalismo se aleja en términos empíricos a lo que sucede dentro de la ZIFEC, su funcionalidad se encuentra ligada a un viejo concepto de integración regional que es lograr un desarrollo económico.

Por otra parte, la segunda etapa, denominada como nuevo regionalismo, se constituye gracias a la inclusión y predominio de epistemologías reflectivistas en su análisis y a los avances tecnológicos que se dieron en el mundo en temas de comunicación y transporte. A causa de esto, una serie de actores no estatales (empresas, movimientos sociales, individuos) y actores subestatales (gobiernos locales, parlamentos o gobiernos autónomos) lograron crear vínculos que trascendían los territorios nacionales en formas nunca antes experimentadas, quitando el monopolio que mantenían los gobiernos centrales sobre el control de las interacciones transnacionales (Matiuzzi de Souza 2018).

En esta línea, Matiuzzi de Souza (2018) propone que la relación entre el nuevo regionalismo y los bordes, tanto en términos teóricos y empíricos, son vistos como una especie de filtro maleable. Por un lado, numerosos eruditos 
predijeron su extinción gracias al análisis del crecimiento de flujos globales de productos, personas e ideas. Mientras que, por otro lado, las fronteras eran vistas como factores claves para la explotación del posicionamiento relativo de ciertos territorios dentro del sistema transnacional en expansión.

En el caso de la ZIFEC se puede observar que existen dos procesos simultáneos de integración fronteriza. Uno impulsado por los Estados con una noción de bordes más estáticos y objetivos guiados desde arriba para lograr un desarrollo ideal. Mientras que existen procesos llevados por los autores no estatales en donde se confluyen una serie de actividades formales e informales que generan una serie de lazos relacionales entre toda la zona fronteriza, la cual es maleable y permeable. En este sentido, la institución informal se encuentra en disputa con la institución formal.

\section{¿Instituciones transnacionales o configuración de preferencias societales?}

Dentro de un mundo interdependiente y globalizado, se comprende que los Estados han cambiado su forma de relacionarse. En el sentido de que el conflicto bélico ya no se configura como el tema principal de la política internacional, sino que se han abierto y masificado los canales de comunicación, cooperación y comercio. De esta manera, se propone que las instituciones internacionales han logrado modificar el comportamiento bélico de los Estados. En el sentido de que generan certezas en un mundo lleno de incertidumbre (Keohane 1988; Stein 2012).

Los canales de cooperación para el desarrollo como el PBIFEC se consolidan como una institución internacional, que se presupone que lograría modificar el comportamiento de los territorios en donde se implemente. No obstante, cuando se plantea dicho concepto dentro de una zona fronteriza, surgen inadecuaciones empíricas, ya que dichas zonas con poca presencia estatal no se han constituido dentro de un proceso normalizado de construcción estatal.

Por otra parte, Andrew Moravcsik (2012), con el llamado nuevo liberalismo, nos plantea un enfoque distinto para analizar los diferentes aspectos que conforman la estructura internacional, enfatizando el rol de las 
relaciones estado-sociedad. Para desarrollar de mejor manera esta visión y vincularla directamente con el tema a tratar, debemos entender entonces, que el análisis es de abajo hacia arriba, lo que quiere decir que los individuos y grupos sociales son actores participes activos del sistema político transnacional.

Por este motivo, es importante partir de una premisa central que Moravcsik plantea, en donde propone que las ideas sociales, los intereses e instituciones influencian en el comportamiento de los estados, y no viceversa. De esta forma, se configuran las preferencias estatales, las cuales son importantes en la política global a partir de intereses individuales. Esta premisa central se sustenta en tres supuestos fundamentales: la primacía de los actores sociales, la representación y preferencias estatales, y la interdependencia y el sistema internacional.

Las unidades societales configuran sus preferencias en pro de los beneficios económicos, políticos o culturales, lo que evidencia que su interés radica en la adversidad al riesgo, y a la vez, esto se justifica por la racionalidad. En otras palabras, mientras menos se arriesga más se gana. Esto, además, está directamente relacionado con el primer supuesto fundamental, puesto que son estos mismos actores los que en busca de su bienestar actúan por medio de acción colectiva e intercambio político.

Este análisis, es claramente visible en las zonas de frontera o zonas grises (Putumayo y Sucumbíos) pues existe, en primer lugar, desconfianza por parte de los habitantes hacia el gobierno central, lo que hace que exista una desconexión estatal. Por esta razón, en estas zonas existen grupos ilegales que tienen mayor influencia que el Estado y generan prácticas cotidianas y beneficios institucionalizados de manera informal consolidados por medio de la costumbre. Sin embargo, existe un interés real por parte de los estados para sobrellevar estas actividades ilícitas. Esto se refleja en el Plan Binacional de Integración Fronteriza, que además de ser este instrumento que genera institucionalidad, ha generado objetivos centrados en diferentes aspectos como lo es la educación, la salud y los servicios básicos entre otros.

El dilema en esto proviene de que los mismos objetivos están siendo propuestos o cumplidos por parte de estos grupos ilegales, además de que hay 
que considerar, en segundo lugar, el factor de bienestar de estos sectores, pues son estos mismos grupos los que generan beneficios, por ejemplo, puestos de empleo y seguridad. Además de que existe una descentralización del Estado, estas zonas han desarrollado cierta autonomía respecto a las agendas de trabajo y el Plan Binacional direccionada al bienestar y libertades de estas zonas (un claro ejemplo es su interés en la maximización de intereses económicos-productivos), lo que hace factible la cooperación con estos grupos informales.

\section{Presentación de resultados y análisis de datos}

Como parte del seguimiento y la evaluación al PBIFEC, el gobierno ecuatoriano con el colombiano implementaron un sistema de información binacional que recoge los datos macro estadísticos que permitan encontrar los efectos que mantienen las políticas conjuntas establecidas en los instrumentos institucionales binacionales dentro de la zona del ZIFEC. En este sentido, se tomaron las estadístics del sistema de información binacional para realizar una evaluación sobre el efecto del PBIFEC. Un primer estadístico es el coeficiente de Gini (Gráfico 1), utilizado con el objetivo de ver las desigualdades basadas en los ingresos que existen en cada departamento o provincia fronteriza y en la ZIFEC en conjunto. Mientras que en el gráfico 2 se puede visualizar las variaciones de datos sobre los embarazos adolescentes de la zona. En el gráfico 3 se visualiza los porcentajes de crímenes violentos. Mientras que los gráficos 4 y 5 muestran variaciones sobre los porcentajes de empleo pleno y precarizado.

\section{Gráfico 1:}

Variación del Coeficiente de Gini. 


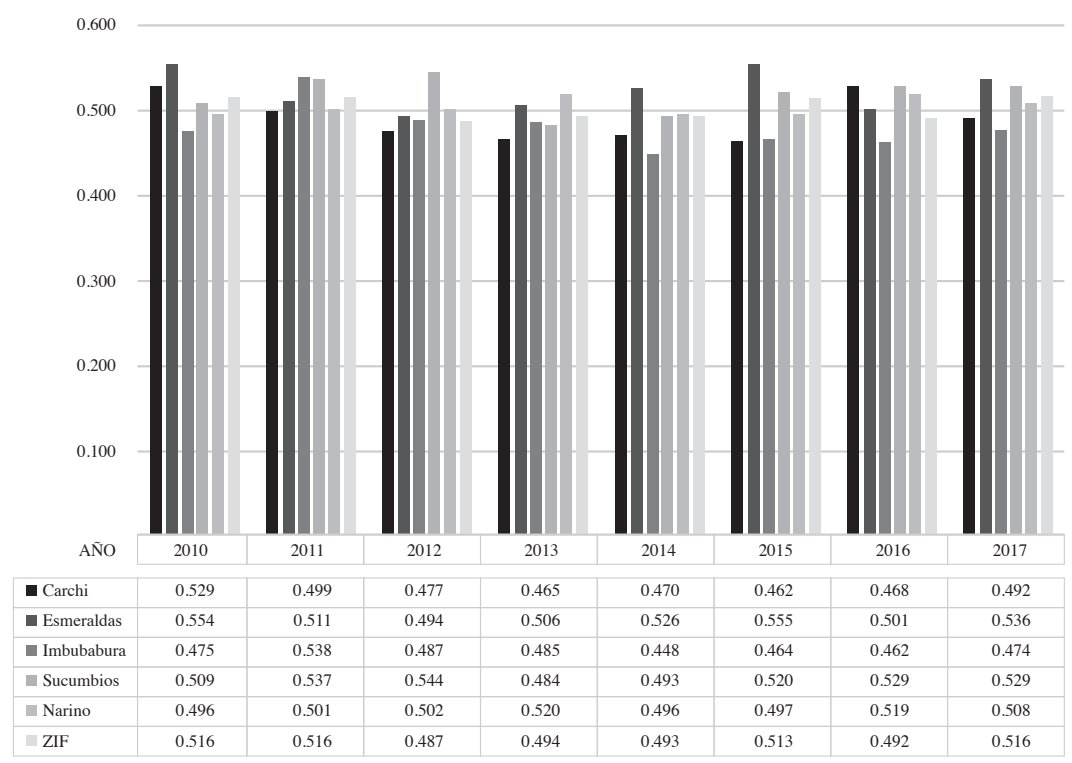

Gráfico elaborado por el autor a partir de los datos de Plan Binacional de Información (2014)

El gráfico 1 demuestra que las desigualdades por ingreso en la zona son similares a los datos regionales. América Latina es una de las regiones mas desiguales del mundo y cuando se descompone dicho coeficiente por subregiones, los resultados van a ser los mismos. En este sentido, las desigualdades percistentes dento de la ZIFEC se han mantenido sin una variación significante. Denotando que el accionar institucional binacional puede cumplir un rol un tanto insignificante en cuanto a la desigualdad estructural, la cual es reflejada en los datos.

\section{Gráfico 2:}

Porcentaje de nacimientos en mujeres de 15 a 19 años. 


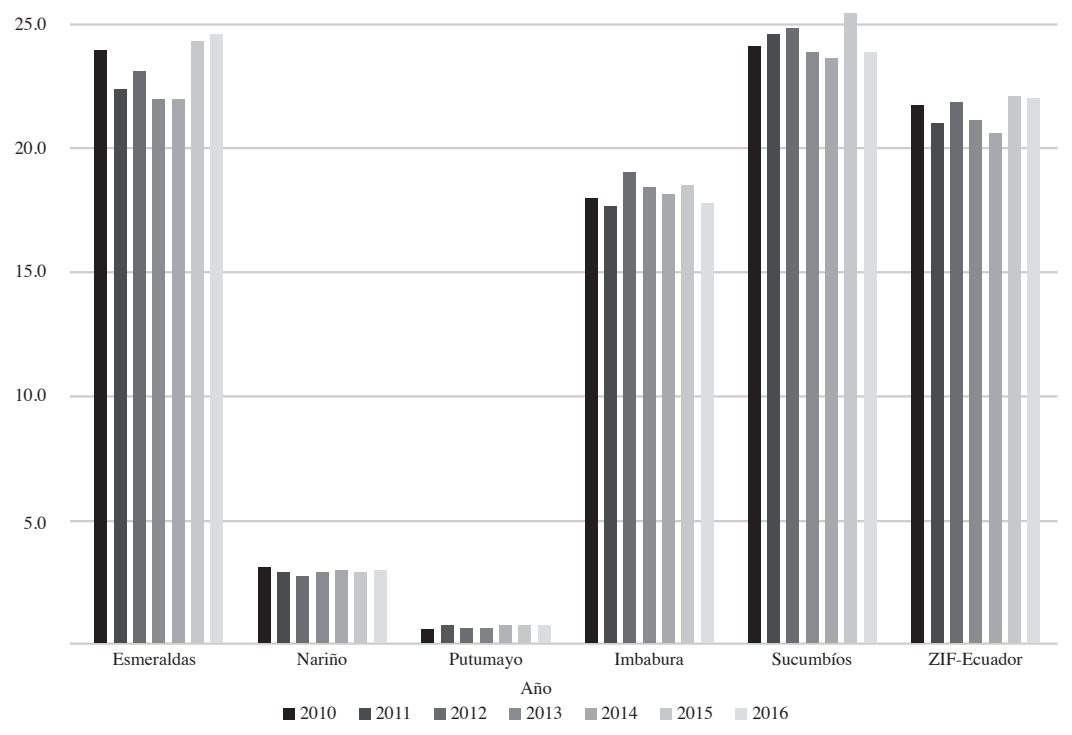

Gráfico elaborado por el autor a partir de los datos de Plan Binacional de Información (2014)

El embarazo en adolescentes es uno de los principales problemas dentro de la sociedad en torno al eje de educación y fortalecimiento social. En Nariño $\mathrm{y}$, especialmente, en Putumayo, se mantiene un registro bajo de la tasa de nacimientos en mujeres de 15 a 19 años en un periodo comprendido desde 2010 a 2016. Caso contrario, en Esmeraldas, Imbabura, Sucumbíos y la zona fronteriza en general, la tasa de embarazos adolescentes es alta, ya que aproximadamente por cada 5 nacimientos, 1 es de una madre adolescente. Si bien, en Colombia esta problemática parece estar controlada, las buenas prácticas para reducir este indicador, al parecer, no se han difundido a las zonas fronterizas ecuatorianas. Como se puede ver en el Gráfico 2, en Sucumbíos $\mathrm{y}$ en Esmeraldas el porcentaje de nacimientos de mujeres adolescentes se ha incrementado, mientras que en los datos de la ZIFEC se ha sostenido y tiene una tendencia al aumento. 


\section{Gráfico 3:}

Porcentaje de crimenes violentos.

35.0

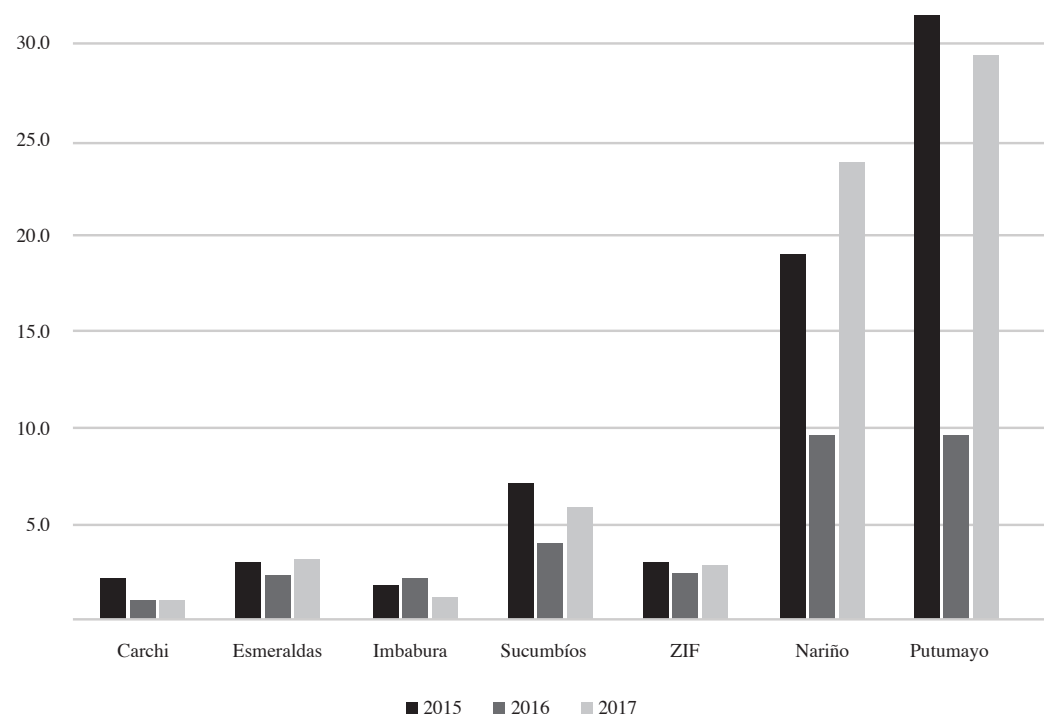

Gráfico elaborado por el autor a partir de los datos de Plan Binacional de Información (2014)

Por su parte, en un periodo comprendido entre 2015 a 2017, la actividad relacionada a crímenes violentos ha sido un detonante a la seguridad que afecta especialmente a Putumayo, alcanzando en 2015 un 32\% de crímenes que utilizan armas de fuego. Esto convierte a este departamento en el que mantiene dicha tasa más alta en comparación con los espacios de las zonas fronterizas ecuatorianas. De igual manera, Nariño mantiene un porcentaje de $29 \%$ en 2017. Sucumbíos, Imbabura, Esmeraldas, Carchi y la ZIFEC mantienen un bajo registro en comparación a las mencionadas anteriormente. Esto denota que los problemas sociales en la zona colombiana están relacionados con cuestiones de violencia, mientras que en Ecuador, una grave problemática es el tema de los embarazos adolescentes como se lo analizó en la anterior grá- 
fica. Ambos temas no han podido ser solventadas por medio de los esfuerzos institucionales binacionales.

\section{Gráfico 4:}

Tasa de empleo adecuado

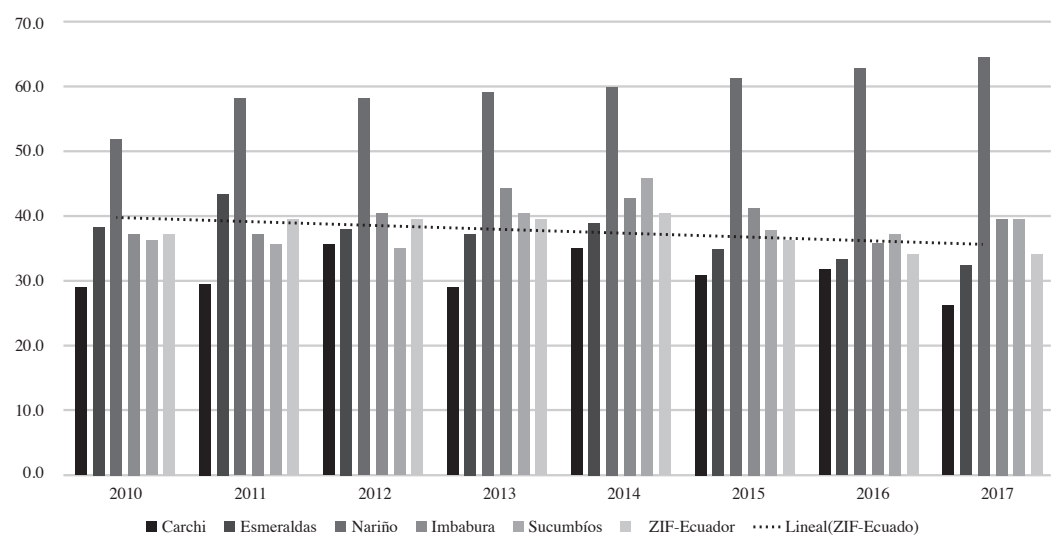

Gráfico elaborado por el autor a partir de los datos de Plan Binacional de Información (2014)

Cuando se analiza los temas relativos a la ocupación, se puede visualizar que Nariño mantiene un registro constante y elevado de empleo adecuado durante el periodo de 2010 a 2017. Denotando el desarrollo productivo del departamento en comparación a los demás. Por su parte, en la zona de Ecuador se puede ver un incremento en el porcentaje de empleo adecuado durante el periodo de 2010 hasta 2014, mientras que empieza a reducirse desde 2015 a 2017. 


\section{Gráfico 5:}

Tasa de empleo no adecuado

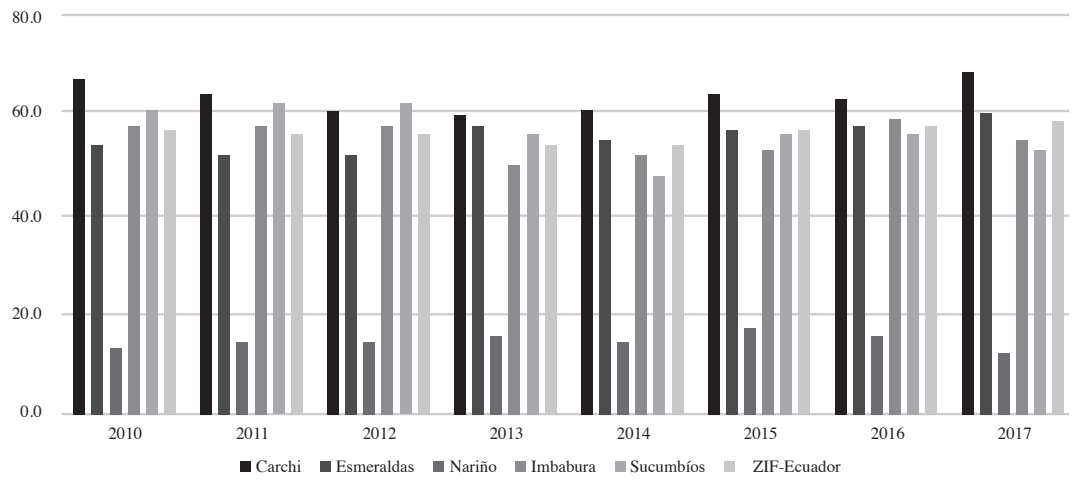

Gráfico elaborado por el autor a partir de los datos de Plan Binacional de Información (2014).

En este mismo sentido, el gráfico 5 que expone datos sobre el empleo precarizado demuestra que Carchi es el sector que presenta la más alta tasa de empleo no adecuado. Seguido no tan de lejos por provincias como Sucumbíos y Esmeraldas. Así, se puede ver que el desarrollo productivo en la ZIFEC no ha mantenido variaciones durante la época de implementación de las políticas binacionales. Esto demuestra que se realizó un esfuerzo conjunto por identificar las problemáticas de la zona, pero, al momento de aplicar las políticas para dar solución a dichas problemáticas, estas no pudieron cumplir con el fin mencionado.

\section{Construcción de institución internacional}

Como comentan los entrevistados E1 y E3, las relaciones entre Ecuador y Colombia, por ser países fronterizos, son muy dinámicas, amplias y abarcan un gran número de temáticas. En este sentido, la filosofía que está detrás de los Gabinetes Binacionales es la de juntar a las personas responsables de los diferentes temas para que dialoguen, se pongan de acuerdo, revisen los proyectos, y pongan en marcha los proyectos que se han establecido en el 
marco de los Gabinetes Binacionales. De esta manera, se genera estrategias de coordinación nacional basadas en un interés común que se lo visualiza en las instituciones binacionales formales como el PBIFEC.

No obstante, con la presencia de grupos y organizaciones ilegales en la ZIFEC, la presencia institucional de los Estados ha sido de poca relevancia. Durante décadas, las actividades realizadas en las fronteras han sido muy desconocidas y han pasado desapercibidas incluso por los gobiernos. La falta de institucionalidad, cuerpos legales y un correcto reclamo de la soberanía ha provocado que las actividades ilegales se tomen estos espacios para la contribución de una economía ilegal basada en la vulneración de derechos humanos e injusticia social (Carrión 2011). En este sentido, los cuatro entrevistados del sector gubernamental (E1, E2, E3 y E4) concuerdan que uno de los temas de mayor relevancia en la ZIFEC tendría que ser las políticas para enfrentar consecuencias de los actos ilegales en ambos lados de la frontera, en lo que tiene que ver con narcotráfico, cultivos ilícitos y comercio informal.

En la frontera norte ecuatoriana se ha podido connotar un auge de la economía fronteriza, la cual ha generado inequidades sociales, procesos migratorios, crecimiento de la violencia y enriquecimiento del crimen organizado. Fernando Carrión (2011) comenta que la economía fronteriza desarrolla una atracción poblacional que minimiza el riesgo para la vida, gracias al crecimiento económico. De esta manera, también se puede denominar a este fenómeno como economía de enclave, pero con la variable de que es un sistema complejo diversificado de intercambios comerciales legales e ilegales que tienen enlaces superiores a los territorios transfronterizos. Esta dinámica es conocida por los funcionarios estatales, por lo que se propuso dentro del plan mejorar las actividades productivas dentro de las zonas, aprovechando las ventajas comparativas de cada sector. Por ejemplo, potenciar el turismo en la zona de Esmeraldas y el Carchi fue la propuesta de los entrevistados E2 y E4.

En este sentido, las necesidades identificadas dentro de la ZIFEC por los funcionarios gubernamentales son claramente las de desarrollo productivo y seguridad. El entrevistado E1 señala la forma en la que se construyeron los ejes de desarrollo multidimensional del PBIFEC con la siguiente frase: 
"La seguridad es un requisito para el desarrollo, por lo que es imposible tener desarrollo sin seguridad, pero también no es fácil tener seguridad sin desarrollo. Las dos cosas se complementan perfectamente".

Otro tema que sale a la luz es el eje social. El entrevistado E3 planteó un ejemplo que permite visualizar los efectos sociales dentro de la ZIFEC. El señaló que existen muchos niños y adolescentes que son tentados por grupos subversivos, ya que ellos pueden ofrecer la seguridad que el Estado no ha sido capaz de brindar. En este aspecto, se planteó la generación de un eje social relacionado con la educación y la protección del tejido social de la zona. Se puede visualizar que Ecuador y Colombia tienen problemas sociales de distinta naturaleza. En Colombia se puede observar que existen expresiones de violencia más naturalizadas, reflejadas en el número de crímenes violentos hacia las mujeres y el número de crímenes violentos que utilizan armas de fuego. Mientras que en Ecuador, uno de los principales problemas son los embarazos adolescentes no deseados.

\section{Construcción de institución informal (preferencias societales)}

La construcción de mercados ilegales se ha percibido como una causa fundamental en el abastecimiento de las ciudades fronteras. La libre comercialización de drogas y armas, así como la de mercados informales de artículos lícitos, han forjado que el derecho a la protección sea un bien que debe ser pagado y respaldado por los mismos ciudadanos marginados por su propio gobierno. Pese a esto, la economía en las fronteras genera un crecimiento de poblaciones ilegales que construyen ciudades frontera, lo que puede dar paso al contrabando, crimen organizado, y actos delictivos condenados por el sistema internacional pero beneficioso para quienes son parte de este sistema. Así, la presencia del Estado como institución se vuelve precaria. El orden institucional del Estado queda limitado y las políticas de acción en materia de seguridad varían en torno a la mitigación de los impactos, algo que es contraproducente, ya que el dinamismo que existe en la frontera suele superar fácilmente la rigidez de las instituciones estatales (Llugsha G. 2015).

Esto puede verse reflejado en los comentarios del F1, donde se declaró 
que no existe confianza de las poblaciones de la ZIFEC en las instituciones gubernamentales. Mientras que la confianza hacia los organismos no gubernamentales, como ACNUR, UNICEF y Cruz Roja se ha ido fortaleciendo. Esto puede ser observado dentro del trabajo entre la sociedad civil y actores no gubernamentales expresado en las reuniones semanales en el centro de atención binacional fronterizo. En donde se plantean soluciones en conjunto con todas las agencias que participan en monitoreo y trabajo de asistencia humanitaria, prescindiendo de la coordinación o presencia de funcionarios estatales. Esto demuestra que los grupos societales de la zona prefieren coordinar directamente las acciones entre ellos sin la necesidad de involucrarse en procesos estatales, lo que genera una institución informal más influyente que la que pueden crear las coordinaciones estatales.

Dentro del F1 se logró corroborar una práctica social institucionalizada de manera consuetudinaria dentro de la comunidad. En Farfán, una comunidad fronteriza llena de asentamientos informales, hay la costumbre de integrar de manera casi automática a cualquier extranjero (distinguible solamente por su acento) dentro de la sociedad. En esta zona, las personas nacionales y extranjeras mantienen una convivencia sin ningún tipo de discriminación ni distinción de nacionalidades, en donde no existe ningún requisito para poder ser aceptados. Por el contrario, en las instituciones gubernamentales y no gubernamentales si existe una distinción entre quienes llegan a dicha zona como migrantes, en donde se requiere solicitarles sus documentos de identificación para cumplir con protocolos internacionales y solamente a partir de dicha distinción ofrecerles ayuda humanitaria o cualquier otro servicio.

Una de las principales razones por las cuales se identifica que la sociedad rechaza estas instituciones binacionales es debido a que, dentro de su gestación, existió un proceso de participación ciudadana mediante las mesas técnicas binacionales, en donde fueron llamados personas de la sociedad civil para establecer las diferentes problemáticas de la ZIFEC. No obstante, F1 comentó que al formar parte de estas mesas técnicas no fueron tomados en consideración varios de los problemas que la sociedad civil mencionó cuando se realizaron los ejes de desarrollo multidimensional. Por ello, el Plan Binacional y Agenda binacional de Sucumbíos y Putumayo nace en base a estos problemas que no fueron considerados. En este caso, las unidades societales 
promovieron sus propias soluciones en base a un método participativo interno.

Todas estas prácticas no son concordantes con lo que han planteado los funcionarios E2 y E4, quienes proponen que los convenios ya se han firmado, los acuerdos se han ratificado, el protocolo ya se ha hecho y ya ha salido en los medios de comunicación. Ahora, la manera que hay que efectivizarlo es por medio de la socialización. En este sentido, las prácticas cotidianas de la ZIFEC, difícilmente pueden ser transformadas por medio de netamente la imposición gubernamental y la socialización de esta. Ya que como se pudo corroborar con las experiencias del F1, hay redes sociales e instituciones informales centenarias que han condicionado las prácticas de la zona. Las cuales solamente pueden ser modificadas por medios de un trabajo a largo plazo que prepondere la presencia prolongada del Estado en estas zonas.

\section{Conclusiones}

El Plan Binacional de Integración Fronteriza es un mecanismo de acción integral y conjunta que nació como fruto de las relaciones bilaterales sostenidas entre Ecuador y Colombia bajo los parámetros establecidos en los Gabinetes Binacionales. Claramente, de cada lado, existe un esfuerzo institucional que prevé generar y promover el desarrollo productivo, económico y social en la ZIFEC, además que marcan un punto de partida para las demás zonas de integración fronteriza que tienen ambos países. En este sentido, se puede visualizar que se generó una institución binacional que sí modificó el comportamiento estatal. Efectivamente, se lograron identificar problemas conjuntos y generar políticas por medio de procesos de cooperación para hacer frente a dichas problemáticas binacionales.

Por su parte, se produjo una homogenización sobre las ideas de desarrollo multidimensional necesarias para reducir la inseguridad, aumentar el desarrollo productivo y consolidar un tejido social en la ZIFEC. Este proceso se logró por medio de lo que se explicó en el anterior apartado, a partir de la identificación de problemáticas comunes que permitieron la elaboración de políticas y acuerdos para consolidar la seguridad y el desarrollo en las zonas de frontera. Actualmente, son más entidades gubernamentales las que trabajan 
alrededor de estas zonas, como el Ministerio de Relaciones Exteriores, SENPLADES, Defensoría del Pueblo, etc. No obstante, la incidencia de estas instituciones ha sido muy baja debido a la falta de confianza que tienen los habitantes de las ZIFEC hacia el gobierno, debido a la poca participación de este durante años y por el nuevo institucionalismo a partir de grupos societales.

Pese a que se ha firmado, ratificado y socializado las instituciones binacionales, estas no han logrado un efecto deseado en ninguno de los tres ejes como lo demuestran las estadísticas. La falta de confianza en las instituciones del gobierno por parte de los habitantes de la ZIFEC ha constituido una nueva institucionalización dentro de estas zonas. Varios grupos societales y habitantes han tomado por su propia mano el desarrollo de esta zona. Pese a la incidencia del gobierno, muchas instituciones se han visto despreocupadas como es el caso de los CEBAF. De esta manera, se puede corroborar que el efecto del PBIFEC no ha conseguido un impacto notable dentro de estas zonas a un nivel poblacional, pese a que en el nivel gubernamental se lograron varios acuerdos.

A pesar que ambos estados han motivado su accionar respecto a problemáticas, no han logrado satisfacer de manera adecuada las demandas locales dentro de la ZIFEC. En este sentido, desde el aspecto social, los habitantes han dado a conocer sus problemas y necesidades, los cuales no se han tomado en cuenta de la manera debida. Las demandas locales van más allá de lo que el gobierno puede ver desde el centro. Finalmente, la teoría nos da paso a entender este fenómeno social desde el aspecto de las Relaciones Internacionales. Morasvick, nos ayuda a comprender, por medio de su presupuesto de aversión al riesgo, las razones por las cuales las poblaciones de las ZIFEC no han recibido bien las instituciones binacionales. Esto debido a: la poca presencia estatal histórica, las prácticas sociales institucionalizadas de manera informal que generan mayores beneficios que los que proponen las nuevas instituciones; una poca incidencia de la participación de las poblaciones en los delineamientos institucionales y mayores beneficios e incentivos que trae el mantener estructuras informales a los que traería las instituciones gubernamentales binacionales pensadas por funcionarios del gobierno central. 


\section{Bibliografía}

Acemoglu, Daron, y James Robinson (2017). Por qué fracasan los países: los orígenes del poder, la prosperidad y la pobreza. Bogotá: Ediciones Deusto.

Arriaga-Rodríguez, Juan Carlos (2011). "El concepto jurídico de frontera". En Memorias del XII Seminario Internacional de Verano. Caribe: Economía, política y sociedad, de Juan Carlos Arriaga-Rodríguez \& T. Camal-Cheluja (Eds.), pp. 471-490. Nicoya: Universidad de Costa Rica.

Caflisch, Lucius (2010). "A Typology of Borders". En International Law: New Actors, New Concepts Continuing Dilemmas, de Budislav Vukas y Trpimir Sosic (Eds.), pp. 183-219. Boston: Brill Publishers.

Carrión, Fernando. (2013). Asimetrías en la frontera Ecuador-Colombia: Entre la complementariedad y el sistema. Quito: FLACSO Serie Fronteras.

CEPAL, N. (2018). Nuevos desafios y paradigmas: perspectivas sobre la cooperación internacional para el desarrollo en transición. Naciones Unidas. Comisión Económica para América Latina y el Caribe (CEPAL), Santiago (LC/PUB.2018/16).

Dai, Xinyuan, Duncan Snidal, y Michael Sampson. (2017). “International Cooperation Theory", International Studies 22: 1-28.

Kaul, Inge, Paul Conceicao, Katell le Goulven y Ronald U. Mendoza. (2003). Providing Global Public Goods: Managing Globalization. Oxford: Oxford University Press.

Matiuzzi de Souza, Gustavo (2018). "Notions of border in regionalism theory and praxis A critical overview" Civitas 18: 245-261. 
Moravcsik, Andrew (2012). "The new liberalism” En The Oxford Handbook of International Relations, de Christian Reus-Smith y Duncan Snidal, 235-266. Oxford: Oxford University Press.

Plan Binacional de Información. Plan Binacional de Información. 2014. http://appdes.senplades.gob.ec/binacional/?page_id=298 (último acceso: 2019).

Prado Lallande, Juan Pablo. (2016). "El liberalismo institucional”. En J. A. Schiavon Uriegas, A. S. Ortega Ramírez, M. López-Vallejo Olvera y R. Velázques Flores (Eds.), Teoría de Relaciones Internacionales en el siglo XXI: Interpretaciones críticas desde México pp. 367-386. México DF: Cide.

Robert, Axelrod, y Robert Keohane (1985). “Achieving Cooperation under Anarchy: Strategies and Institutions”, World Politics 33: 226-254.

Seara Vázquez, Modesto. (2004). Derecho Internacional Público, 20ª ed., México: Porrúa.

Senplades-DNP. Plan Binacional de Integración Fronteriza (2014). Documento Oficial, Quito y Bogotá: Senplades y DNP.

Stein, Arthur (2012). "Neoliberal Institutionalism" En The Oxford Handbook of International Relations, de Christian Reus-Smith y Duncan Snidal, 201-221. Oxford: Oxford University Press. 